\title{
Influence of adsorbing species on properties of equilibrium silver iodide clusters
}

\author{
Ivana Lj. Validzic and Willem K. Kegel * \\ Van't Hoff Laboratory for Physical and Colloid Chemistry, Debye Research Institute, Utrecht University, Padualaan 8, 3584 CH Utrecht, The Netherlands
} Received 31 October 2003; accepted 20 January 2004

Available online 28 February 2004

\begin{abstract}
Thermodynamically stable AgI clusters were studied in the presence of high- and low-molecular-weight additives: polyethyleneimine (PEI) and dimethylformamide (DMF), respectively. Clusters containing up to 20 silver iodide pairs, roughly twice as many as in the system without PEI or DMF, have been observed. We show that the mechanism stabilizing these clusters is mixed adsorption with iodide ions at the AgI-electrolyte interface. We make it plausible that more strongly adsorbing additives give rise to ultralow interfacial tensions of the AgI-electrolyte interface, with perspectives for "reversible colloids."
\end{abstract}

(C) 2004 Elsevier Inc. All rights reserved.

\section{Introduction}

In this work we address the question of what adsorbing additives do to the properties of stable AgI clusters in the presence of excess iodide ions. We recently showed that the insoluble salt silver iodide partly emulsifies into small clusters containing up to $10 \mathrm{AgI}$ pairs if sufficient excess iodide ions are present [1]. The mechanism behind this spontaneous emulsification is that iodide ions adsorb at the interface between solid silver iodide and the aqueous electrolyte solution, thereby decreasing its interfacial tension. This mechanism is fundamentally different from the mechanism that underlies traditional colloid stability: stability of colloids in the traditional sense refers to their ability to remain suspended by avoiding aggregation due to attractive (e.g., van der Waals) components in the potential of mean force between particles [2]. In that case the large interface present in these systems represents a large amount of free energy, which is only at a minimum when all the particles have united into a single large crystal. On the other hand, there is a close analogy with microemulsions, dispersions (on nanometer scales) of immiscible liquids stabilized by surfactant molecules; these form spontaneously once the surface work for creating droplets becomes on the order of the thermal energy—see, e.g., [3].

\footnotetext{
* Corresponding author.

E-mail address: w.k.kegel@chem.uu.nl (W.K. Kegel).
}

However, the interfacial tension of the surfactant-loaded oil-water interface in the case of microemulsions usually is below $0.1 \mathrm{mN} / \mathrm{m}$, while in the systems studied in [1] it was found that the interfacial tension is roughly $10 \mathrm{mN} / \mathrm{m}$. This value is an order of magnitude smaller than the interfacial tension of the bare AgI-water interface, being of order 100 $\mathrm{mN} / \mathrm{m}$ [4]. The larger interfacial tension compared to that of microemulsions leads to relatively small clusters, as the size of a cluster must be on the order of $\sqrt{k T / 4 \pi \gamma}$, where $k T$ is the thermal energy and $\gamma$ the interfacial tension. This equation follows from the condition that (spherical) clusters of radius $R$ must form if the work to create them is on the order of the thermal energy, i.e., $4 \pi R^{2} \gamma \approx k T$.

The purpose of this work is to investigate the influence of adsorbing materials other than iodide and to study the cluster properties. If more than a single species adsorbs at the AgIelectrolyte interface, interfacial tension is expected to decrease even further than in the situation with only iodide, resulting in clusters significantly larger than those found in [1]. Adsorption of these components should still be reversible, as thermodynamic equilibrium requires that exchange of matter between clusters occur.

In this paper both high- and low-molecular-weight adsorbing species are studied. In the next paragraph we study silver iodide in the presence of polyethyleneimine, PEI. These polymers are well known to adsorb onto silver iodide, and they at least kinetically stabilize silver iodide quantum dots [5]. Next we move to $N, N$-dimethylformamide (DMF). 
Silver iodide has a high solubility in this solvent and forms complexes containing up to six silver atoms [6].

\section{Experimental}

\subsection{Materials}

Samples were prepared using distilled water. Silver perchlorate hydrate, silver nitrate, potassium iodide, polyethylenimine (50 wt $\%$ solution in water, $M_{w} \approx 2000$ ), and $N, N$-dimethylformamide were used without further purification.

\subsection{Spectrophotometry}

Optical absorption spectra were recorded using a Cary 1E UV-Vis spectrophotometer. The temperature of the solution was maintained at $25 \pm 0.1^{\circ} \mathrm{C}$. Cells with a $1-\mathrm{cm}$ path length were used. The solution in the blank cell contained potassium iodide and sodium thiosulfate, but no perchlorate and PEI or DMF. Neither perchlorate ions nor PEI (DMF) absorb at the wavelengths observed. Most samples contain excess silver iodide solid; the absorption data were taken from the liquid phases after the solid was removed by centrifugation. All solutions were kept in a darkened cabinet. The absorption was determined at several times after preparation and after the performance of temperature cycles in order to verify that the systems are thermodynamically stable. Sodium thiosulfate was present in both the sample and the blank at approximately $8 \times 10^{-4} \mathrm{M}$. At this concentration it does not contribute to the absorption but keeps iodide in the reduced state.

\subsection{Mass determinations}

The buoyant masses of the complexes were determined in a Beckman XL-I analytical ultracentrifuge. The ultracentrifuge contains a spectrophotometer and the equilibrium optical absorption profile was measured at several wavelengths. It usually took between 10 and 15 days before equilibrium was established.

\section{Results and discussion}

\subsection{Addition of polyethyleneimine}

Our first goal was to check if systems containing only small excess amounts of either silver or iodide ions in the presence of PEI, as studied by Mulvaney [5], are thermodynamically stable. These systems contain quite monodisperse, nanometer-sized particles. We found that they clearly are not thermodynamically stable; the adsorption spectra slowly kept shifting (in a matter of weeks to months) toward the red, pointing to larger and larger particles forming [5]. Only

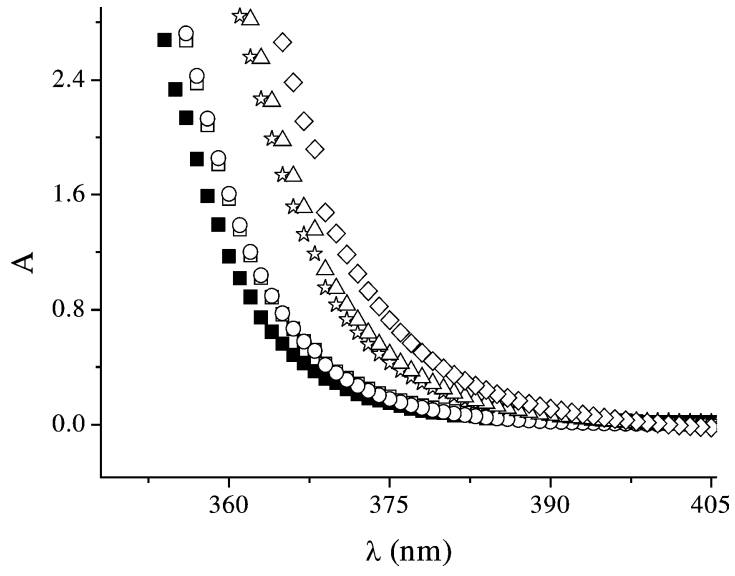

(a)

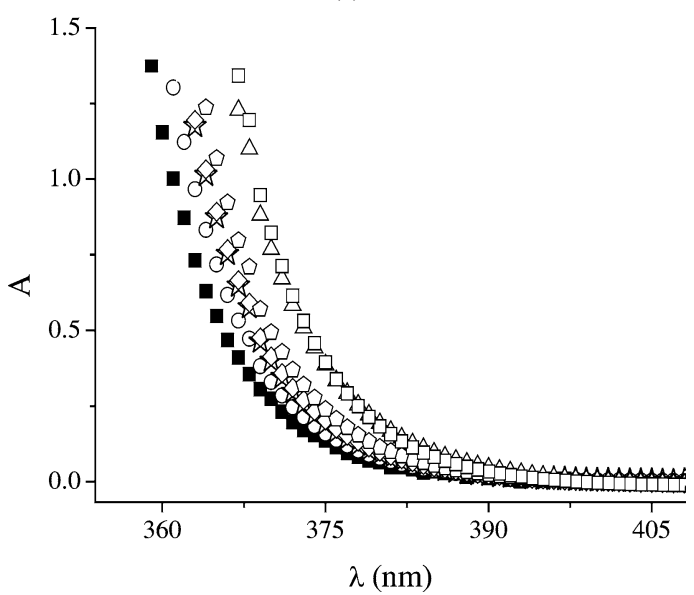

(b)

Fig. 1. (a) Optical absorption of solutions containing $5 \mathrm{M} \mathrm{KI}$ and $1.25 \mathrm{M}$ $\mathrm{AgClO}_{4}(\square)$, plus $5 \mathrm{mM}$ PEI (○); $10 \mathrm{mM}$ PEI ( $\left.\square\right) ; 30 \mathrm{mM}$ PEI (灾); 50 $\mathrm{mM}$ PEI $(\triangle) ; 100 \mathrm{mM}$ PEI $(\diamond)$. These absorptions were measured relative to a system without PEI and $\mathrm{AgClO}_{4}$. A quantity of $8 \times 10^{-4} \mathrm{M}$ sodium thiosulfate was present in the systems in order to avoid formation of $\mathrm{I}_{3}^{-}$ [10]. In these systems an excess solid phase is present. The absorption data were taken from the liquid phases after removing the solid by centrifugation. (b) Same as (a), but now with addition of DMF. Optical absorption of solutions containing $5 \mathrm{M} \mathrm{KI}$ and $1.25 \mathrm{M} \mathrm{AgClO}_{4}$ (ם), plus 0.05 M DMF $(\bigcirc) ; 0.3 \mathrm{M} \mathrm{DMF}(\downarrow)$ ); $0.5 \mathrm{M} \mathrm{DMF}(\diamond) ; 0.8 \mathrm{M} \mathrm{DMF}(\bullet) ; 1 \mathrm{M} \mathrm{DMF}(\triangle)$; 1.5 M DMF ( $\square)$.

systems with compositions of approximately $0.2-0.3 \mathrm{mM}$ PEI, $0.2 \mathrm{mM} \mathrm{AgI}$, and $0.6 \mathrm{mM}$ excess silver ions showed remarkable stability; i.e., no significant red-shift was observed over several months. However, addition of the components in different order revealed no indication that the system containing small particles is thermodynamically stable under those conditions. Indeed, thermodynamic stability implies that system properties depend only upon the thermodynamic state and not, e.g., on the order in which components are added.

The situation is completely different if large excess amounts of iodide are present. Fig. 1a shows absorption spectra of systems containing excess solid AgI after the solid is removed. It is clear that when the polymer concentration is increased in a system containing $5 \mathrm{M} \mathrm{KI}$ and $1.25 \mathrm{M} \mathrm{AgClO}_{4}$ 
a red shift occurs: absorption takes place at increasingly longer wavelengths compared to the system without PEI [1]. The significant red shift with increasing PEI concentration was also observed in a less concentrated system with excess iodide (solution containing $0.57 \mathrm{M} \mathrm{KI}$ and $0.012 \mathrm{M} \mathrm{AgClO}_{4}$ plus different PEI concentration from 0.12 to $20 \mathrm{mM}$; spectra not shown). The observed red shift may occur due to the presence of AgI clusters, stabilized with PEI (besides iodide ions), when the polymer concentration increases. It will be shown later that this is indeed the case. At this point we note that the absorption spectra in Fig. 1 are independent of the order in which the components were added. Moreover, they cannot be distinguished from the original spectra after performing temperature cycles. These observations are strong proof of thermodynamic stability of the systems.

Our next step is to find out what the size (distribution) of the clusters is. We will follow the strategy pointed out in [1], i.e., finding the relation between the size of the particles and the wavelength where they absorb, using ultracentrifugation (UC). In short, for semiconductor particles such as AgI, the band gap energy of an electron-hole pair depends on the size of the particles [7], leading to

$\frac{1}{\lambda}=\frac{1}{\lambda_{0}}+\frac{\alpha}{R^{2}}$,

where $\lambda$ is the absorption wavelength, $R$ is the particle size, $\lambda_{0}=420 \mathrm{~nm}$ is the value of the wavelength where the bulk exciton absorption of $\mathrm{AgI}$ is centered [8], and $\alpha$ is a constant that can be determined by (UC), at least in principle [1].

For this purpose we measured the optical absorption profile as a function of wavelength, using UC. For an ideal, noninteracting single-component system, the equilibrium distribution obtained is a well-known exponential function of the buoyant mass of the macromolecule, $m_{b}$, given by

$c(r)=c\left(r_{0}\right) \exp \left(\frac{m_{b} \omega^{2}\left(r^{2}-r_{0}^{2}\right)}{2 k T}\right)$,

where $c(r)$ is the sample concentration at radial position $r, c\left(r_{0}\right)$ is the sample concentration at a reference radial distance $r_{0}, \omega$ is the rotational velocity, and $k$ and $T$ are Boltzmann's constant and the absolute temperature, respectively. The optical absorption profile is $A(r)=\varepsilon l c(r)$, where $c(r)$ is given by Eq. (2) and $\varepsilon$ and $l$ are the (molar) extinction coefficient and the optical path length, respectively. If several different species are present, they will, depending on their size, absorb at different wavelengths. Different wavelengths will pick up different sizes and thus different buoyant masses, $m_{b}$ in Eq. (2). As can be seen in Fig. 2a, this is indeed what happens: the longer the wavelength, the larger the buoyant mass of the particles. The real masses of the particles that can be extracted from Fig. 2a correspond to an equivalent number of AgI pairs in the cluster between $n=8.4$ and 11.9, assuming that clusters of size $n$ only absorb at a single wavelength. Compared with the AgI nanoparticles without PEI [1], where clusters of 2.8-6.2

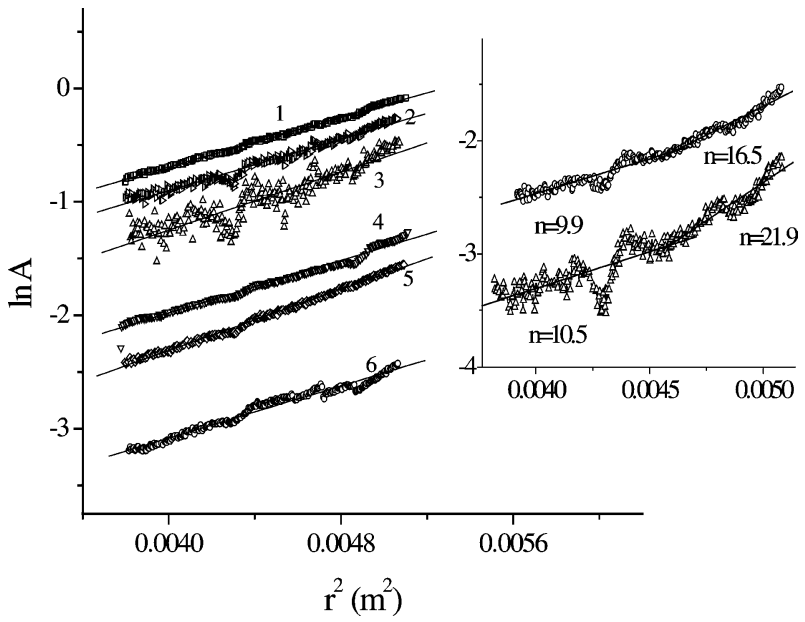

(a)

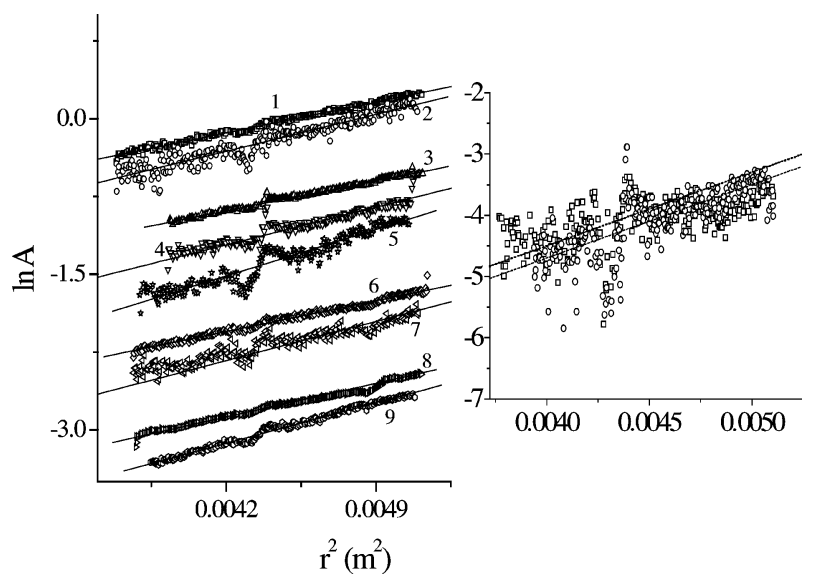

(b)

Fig. 2. (a) Equilibrium optical absorption profiles of systems with different PEI concentrations and constant concentrations of iodide and silver (5 M KI and $\left.1.25 \mathrm{M} \mathrm{AgClO}_{4}\right)$ in an ultracentrifuge $(\omega=12 \mathrm{krpm})$, plotted as $\ln A$ versus $r^{2}$ (see Eq. (2) and the discussion following it in the text). The wavelength-dependent buoyant masses, $m_{b}$, of the particles that follow from the slopes of the curves are expressed in units of $n$, the equivalent number of AgI pairs. These numbers are $n=8.4$ (365 nm; $10 \mathrm{mM} \mathrm{PEI):} \mathrm{curve} \mathrm{(1)} \mathrm{in}$ the figure, $n=9.6$ (375 nm; $10 \mathrm{mM} \mathrm{PEI):} \mathrm{curve} \mathrm{(2),} n=11.9(380 \mathrm{~nm} ; 10$ mM PEI): (3), $n=9.7$ (370 nm; $50 \mathrm{mM}$ PEI): (4), $n=10.4$ (380 nm; 50 mM PEI): (5), and $n=9.3$ (380 nm; $100 \mathrm{mM}$ PEI): (6). Inset: equilibrium optical absorption profiles of systems with $50(\bigcirc)$ and $100 \mathrm{mM}(\triangle)$ PEI at $390 \mathrm{~nm}$. The curves are noisy (and seem to consist of two linear parts) due to very small absorption on the observed wavelength (see explanation above). The number of AgI pairs calculated from the slopes and indicated in the figure is $n \approx 20$. (b) As (a), but now for systems with different DMF concentrations. The masses in equivalent numbers of AgI pairs are: $n=7.1$ (370 nm; 0.05 M DMF): curve (1), $n=8.4$ (380 nm; 0.05 M DMF): (2), $n=6.9$ (370 nm; 0.3 M DMF): (3), $n=8.7$ (380 nm; 0.3 M DMF): (4), $n=11.9$ (390 nm; 0.3 M DMF): (5), $n=7.2$ (370 nm; 0.5 M DMF): (6), $n=10.1$ (380 nm; 0.5 M DMF): (7), $n=8.2$ (370 nm; $1.5 \mathrm{M} \mathrm{DMF):} \mathrm{(8),}$ and $n=11.6$ (380 nm; 1.5 M DMF): (9). Inset: equilibrium optical absorption profiles of systems with 0.5 and $1.5 \mathrm{M} \mathrm{DMF}$ on $390 \mathrm{~nm}$. The masses that follow from the slopes are $n=21.9$ (390 nm ( $\square)$; $0.5 \mathrm{M} \mathrm{DMF)} \mathrm{and}$ $n=21.2(390 \mathrm{~nm}(\mathrm{O}) ; 1.5 \mathrm{M} \mathrm{DMF})$.

were found, it is clear that the clusters are larger in the presence of PEI. If the absorption intensity became too small the curves are noisy (inset, Fig. 2a) and it is difficult to extract 
reliable masses, due to a combination of a small optical path length of the cell that was used in the UC measurements and the limited sensitivity of the optical detector of the UC. However, the number of AgI pairs obtained from the slope in the inset in Fig. 2a is $n \approx 20$.

As can be seen in Fig. 2a (inset), the curves at the highest measured wavelengths seem to consist of two linear parts, pointing to two different-sized clusters picked up by these wavelengths. We return to this issue later. We have also measured the optical absorption profiles as a function of wavelength for $0.57 \mathrm{M} \mathrm{KI}$ with different concentrations of PEI. The sizes of the clusters, expressed in number of AgI pairs are between 7.7 and 10.9. There is no big difference in masses of the clusters found in 0.57 and $5 \mathrm{M} \mathrm{KI}$, although in $0.57 \mathrm{M} \mathrm{KI}$ we found no direct indications that clusters of sizes around $n=20$ exist.

In Fig. 2 rotational velocities were relatively low, up to $12 \mathrm{krpm}$. Around that value, observed particle sizes do not depend on rotational speed, indicating that the centrifugal field does not perturb the cluster distributions present in weak fields (such as under normal gravity). At higher speeds, the situation is quite different: Fig. 3 shows equilibrium optical absorption profiles of systems rotated at 15 and $17 \mathrm{krpm}$. As can be seen in Fig. 3, the buoyant masses (which have been extracted from the steepest parts of the curves for the system observed at $15 \mathrm{krpm}$ ) of the complexes are much larger at these higher rotational velocities than they are in Fig. 2a, reaching values as large as $121 \mathrm{AgI}$ pairs. This indicates that particles tend to coagulate due to the increased rotational velocity. That this scenario applies rather than the size distribution shifting continuously to larger particle sizes has been corroborated by close inspection of the complete absorption profiles at $15 \mathrm{krpm}$ : these profiles can be well described by two linear parts, as shown by the curves designated "a" in Fig. 3. The two masses extracted from these profiles correspond to the two sizes that are found by separate centrifugation experiments at relatively low speeds and at relatively high speeds. At the even higher speed of $17 \mathrm{krpm}$ (curves b in Fig. 3), the only reliable part in the equilibrium absorption profile corresponds to the highest mass clusters. These effects have not been observed for comparable systems without PEI.

We now have all the information to extract the size distribution of the complexes from the optical absorption spectra following [1]. According to Eq. (1), plotting $1 / \lambda-1 / \lambda_{0}$ versus $n^{-2 / 3} \propto R^{-2}$ gives a straight line with a slope related to the value of the constant $\alpha$. Our results are consistent with the value of $\alpha=(0.82 \pm 0.03) \times 10^{-4} \mathrm{~nm}$ we found for the systems without PEI; see [1]. We extract the number of AgI pairs, $n$, from the wavelength by writing (again, see [1] for details)

$n(\lambda)=\frac{4 \pi}{3 v} \alpha^{3 / 2}\left(\frac{1}{\lambda}-\frac{1}{\lambda_{0}}\right)^{-3 / 2}$,

with $v=0.07 \mathrm{~nm}^{3}$, the molecular volume of $\mathrm{AgI}$ in the thermodynamically stable solid $\beta$ (wurtzite) phase.

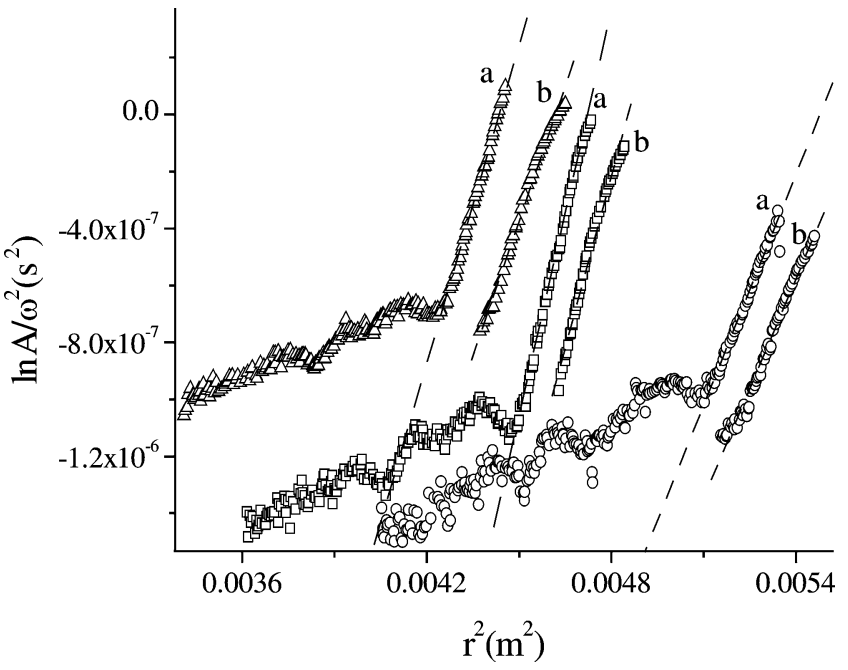

Fig. 3. Equilibrium optical absorption profiles of systems with two different PEI concentrations and constant concentrations of silver and iodide (5 M KI and $\left.1.25 \mathrm{M} \mathrm{AgClO}_{4}\right)$ in an ultracentrifuge plotted as $\ln A / \omega^{2}$ versus $r^{2}$. The solutions contain $10 \mathrm{mM}$ PEI, $n=69(385 \mathrm{~nm}, 0) ; 100 \mathrm{mM}$ PEI, $n=95$ $(390 \mathrm{~nm}, \triangle$ ) and $100 \mathrm{mM}$ PEI, $n=121(400 \mathrm{~nm}, \square)$. The three series of curves represent the profiles of the clusters at 15 (a) and 17 (b) krpm. At higher speed the buoyant masses of the complexes clearly are much larger (compare $m_{b}$ at 12,000 (Fig. 2a) and 15,000 r.p.m.), indicating that particles coagulate due to the increased rotational velocity. At the lowest rotational speeds, the curves (designated (a)) seem to consist of two linear parts.

In Appendix A we analyze the equilibrium

$n \mathrm{AgI}(\mathrm{s})+m \mathrm{I}^{-}+p \mathrm{PEI} \rightleftarrows \mathrm{Ag}_{n} \mathrm{I}_{n+m}^{m-}(\mathrm{PEI})_{p}$,

where $\mathrm{AgI}(\mathrm{s})$ is the (macroscopic) solid, undissolved aggregate of silver iodide. This analysis leads to the size distribution of the clusters

$c_{n}=b e^{-a n^{2 / 3} / k T}$,

where $c_{n}$ is the (molar) concentration of clusters containing $n \mathrm{AgI}$ pairs and in principle, every possible number of excess iodide and PEI. In the absence of PEI we showed that the dependence on $n$ of the first term on the rhs of Eq. (5) is weak [1] compared to the exponential dependence in the second term. The exponential term of Eq. (5) contains the interfacial free energy of the cluster. In the case of spherical clusters with a radius $R$ we have $a n^{2 / 3}=4 \pi R^{2} \gamma$, with $\gamma$ the interaction free energy per unit area. This quantity can be considered as the macroscopic interfacial tension between the excess solid and the electrolyte solution in the limit where that clusters become macroscopically large. The size distribution of the clusters may now be deduced from the absorption spectrum: the relation between $n$ and $\lambda$ is given by Eq. (3), and the relation between $c_{n}$ and optical absorption by the Lambert-Beer law. By plotting the (logarithm of the) optical absorption as a function of $n^{2 / 3}$ for three systems with constant concentrations of iodide and silver, but varying PEI concentrations (10, 50, and $100 \mathrm{mM} \mathrm{PEI),} \mathrm{the} \mathrm{interfacial}$ tension can be estimated. Compared with the system without PEI (see Ref. [1]), the interfacial tension decreases. For $5 \mathrm{M} \mathrm{KI}$ without PEI the interfacial tension was $9.3 \mathrm{mN} / \mathrm{m}$ 


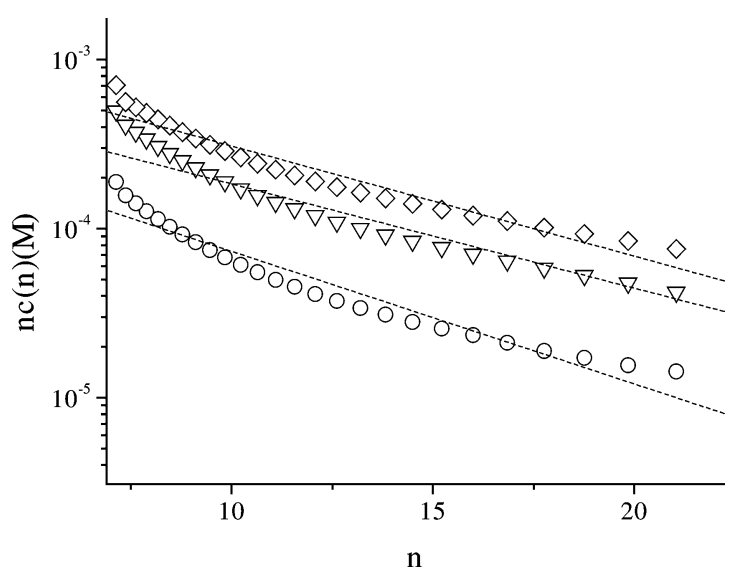

(a)

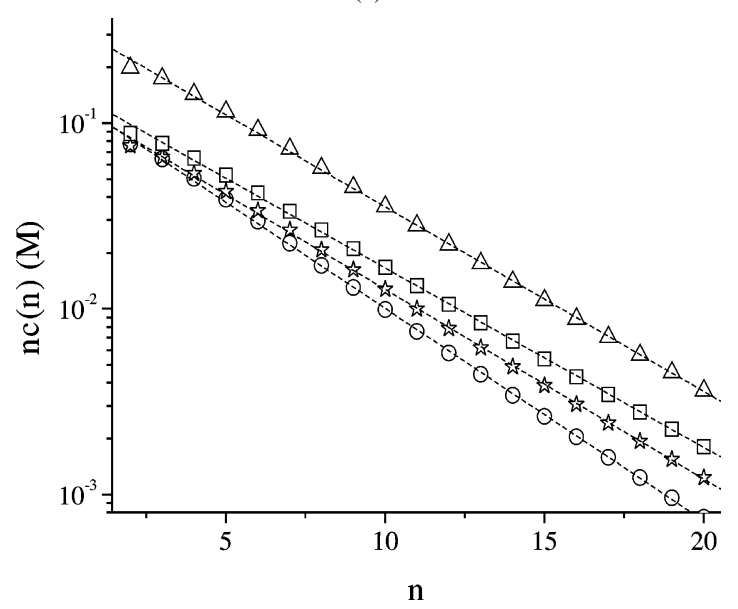

(b)

Fig. 4. (a) Concentration of $\mathrm{AgI}$ in clusters of size $n, n c_{n}$, with $c_{n}$ given by Eq.(5). The values of $b$ were determined as explained in Appendix B. The systems contained $10(\bigcirc), 50(\nabla)$, and $100(\diamond) \mathrm{mM}$ PEI, respectively. As can be seen, the systems contain a significant fraction of silver in clusters with $n \geqslant 20$. (b) As (a), but now the values of $b$ were determined by measuring the mass of the excess phase (explained in Appendix B). They are $b=0.26,0.22,0.24$, and $0.56 \mathrm{M}$ for the systems containing $0.05(\mathrm{O})$, $0.3($ 污), $1.5(\square)$, and $0.5(\triangle)$ M DMF, respectively.

and in the presence of PEI it is $5.5 \mathrm{mN} / \mathrm{m}(10 \mathrm{mM}$ PEI), $5.2 \mathrm{mN} / \mathrm{m}(50 \mathrm{mM}$ PEI), and $4.8 \mathrm{mN} / \mathrm{m}(100 \mathrm{mM} \mathrm{PEI})$ (the values of $a / k T$ are $1.089,1.032$, and 0.951 , respectively). It seems that the value of $\gamma$ only weakly depends on PEI concentrations. Judging from the slight systematic deviation of the data from the theoretical lines in Fig. 4a, it is concluded that either the interfacial tension or the value of $b$ or both slightly depends on $n$. With the low interfacial tensions that we find, clusters containing up to $20 \mathrm{AgI}$ pairs are formed, as can be seen in Fig. 4a.

\subsection{Addition of dimethylformamide (DMF)}

Optical absorption spectra of a system containing $5 \mathrm{M}$ $\mathrm{KI}, 1.25 \mathrm{M} \mathrm{AgClO}_{4}$, and several amounts of added DMF are shown in Fig. 1b. Clearly the same trend is observed as with PEI: with the increasing amount of DMF in the system, a clear red shift occurs; absorption takes place at longer wavelengths compared to the system without DMF. This must be caused by AgI clusters stabilized by DMF/excess iodide. We also found that when the DMF concentration increases, the total concentration of silver in solution significantly increases. In the case of systems containing 0.5 and 0.8 M DMF, no excess phase is present and all silver $(1.25 \mathrm{M})$ dissolves. When the DMF concentration is even further increased, however, excess AgI reappears. This behavior is similar to that of microemulsions, where salt or cosurfactant increases the curvature of the microemulsion drops, which ultimately expel part of their interiors as a macroscopic excess phase [3].

We performed UC measurements to obtain the optical absorption profile as a function of wavelength (see previous section for details). The results are presented in Fig. 2b. In fact, the concentration of clusters in solution is too high to ignore interactions between them (see also Fig. 4b and Appendix B), but clearly no systematic deviations from an exponential profile can be observed. As can be seen in Fig. 2b, the larger the wavelength the larger the buoyant mass of the particles, just as in the case with PEI. The sizes of the clusters as extracted from Fig. 2b correspond to between 7.1 and 18 (inset) equivalent number of AgI pairs.

The value of $\alpha=(0.72 \pm 0.13) \times 10^{-4} \mathrm{~nm}$ that we extract from the data (in the same way as in the previous section) is consistent with the value of $\alpha=(0.82 \pm 0.03) \times 10^{-4} \mathrm{~nm}$ found earlier [1]. Using this value in Eq. (3) we immediately obtain the size distribution and extract the interfacial tensions. For $5 \mathrm{M}$ KI without DMF the interfacial tension was found to be $9.3 \mathrm{mN} / \mathrm{m}$ and in the presence of DMF it is 6.0 , $5.6,5.5$, and $5.3 \mathrm{mN} / \mathrm{m}$ for the systems containing $0.05,0.3$, 0.5 , and $1.5 \mathrm{M} \mathrm{DMF}$, respectively.

In Appendix B we describe how the values of $b$ in Eq. (5) were obtained. With these values, the cluster size distribution, Eq. (5), is now fully determined. In Fig. 4b we plotted the concentration of AgI pairs in clusters of size $n, n c_{n}$. As can be seen in this figure, the systems contain a significant fraction of silver in the clusters. Complexes with sizes comparable to those with PEI form (i.e., up to $20 \mathrm{AgI}$ pairs).

In ending this section we note that we also performed cryo-electron-microscopy measurements on our samples (not shown). We indeed observed clusters with sizes of one to several nanometers, but it has not been possible to measure the sizes more accurately, probably because of the high concentration of silver in solution: 1.25 M. The sizes of the clusters are in that case smaller than or equal to the distances between them. It is worth mentioning, however, that the particles were observed to coagulate on the (charged) carbon surface of the sample holder, which may have applications in, e.g., catalysis.

\section{Concluding remarks}

In this work we showed that if adsorbing species other than iodide (or, more generally, potential-determining ions) 
are present, AgI clusters roughly twice as large as those without additives form. In addition, the whole distribution of clusters shifts (vertically in Fig. 4) toward (up to 1000 times) higher concentrations. The larger clusters form because the interfacial tension of AgI electrolyte is reduced by roughly a factor of 2 compared to the situation without additives (other than iodide_-see Ref. [1]), as prescribed by Eq. (5). The shift of the whole distribution is quantified by a larger prefactor, $b$, in Eq. (5). These observations constitute a proof of principle that even larger clusters can be formed if the tension of the AgI-electrolyte interface is further reduced. This may be realized by more strongly adsorbing species than the ones studied here. We also mention that in order to obtain a narrow size distribution of clusters around a value of, say, several nanometers, the simplest possible scenario requires the interfacial tension to quadratically depend on the size of the clusters [9]. The quadratic well should have a minimum corresponding to a size of several nanometers. This is the case in microemulsions [3]. Such an elastic contribution may in principle be realized if adsorbing species interact, either by attraction or by repulsion, as in surfactant systems.

\section{Appendix A. Multichemical equilibrium and cluster size distribution}

Clusters of silver iodide stabilized by PEI (polyethylenimine) or by DMF-water mixture in the presence of excess $\mathrm{I}^{-}$are in equilibrium with a solid phase of $\mathrm{AgI}$ described by

$n \operatorname{AgI}(\mathrm{s})+m \mathrm{I}^{-}+p \mathrm{Q} \rightleftarrows \operatorname{Ag}_{n} \mathrm{I}_{n+m} \mathrm{Q}_{p}$,

where $\operatorname{AgI}(\mathrm{s})$ is the (macroscopic) solid, undissolved aggregate of silver iodide, $\mathrm{I}^{-}$is the iodide ion, $Q$ is PEI or DMF, and $\operatorname{Ag}_{n} \mathrm{I}_{n+m} \mathrm{Q}_{p}$ is a cluster containing $n$ silver iodide pairs, $m$ excess iodide ions, and $p$ molecules of PEI or DMF.

Thermodynamic equilibrium implies that $\sum_{i} v_{i} \mu_{i}=0$, where $\nu_{i}$ and $\mu_{i}$ are the stoichiometric coefficients and the chemical potentials of the components $i$, respectively. The chemical potentials of the soluble species depend on concentration. For $i=\mathrm{Q}$ and $i=\mathrm{I}^{-}$we write the chemical potential as $\mu_{i}=\mu_{i}^{0}+k T \ln \left(f_{i} c_{i}\right)$, where $\mu_{i}^{0}$ is the standard chemical potential, $c_{i}$ the concentration of $i$ relative to the "standard concentration" $c^{0}=1 \mathrm{M}$, and $f_{i}$ is the activity coefficient. Similarly, for $\operatorname{Ag}_{n} \mathrm{I}_{n+m} \mathrm{Q}_{p}$ we write the chemical potential as

$\mu_{n, m, p}=\mu_{n, m, p}^{0}+k T \ln x_{n, m, p}$,

where the subscript $(n, m, p)$ refers to the cluster $\operatorname{Ag}_{n}$ $\mathrm{I}_{n+m} \mathrm{Q}_{p}$. The logarithmic term in the above equation now contains the mole fraction $x_{n, m, p}$ instead of the (molar) concentration. We neglect deviations from ideal behaviour of the clusters, although the concentrations of species in solution are not small. In dispersion, the clusters are surrounded by electrolyte solution and PEI or DMF rather than being part of a lattice. This gives rise to an interfacial free energy term in the standard chemical potential of a cluster. We therefore write

$\mu_{n, m, p}^{0}=n \mu_{\infty}^{0}+m \mu_{\mathrm{I}^{-}}^{\mathrm{ins}}(n)+p \mu_{\mathrm{Q}}^{\mathrm{ins}}(n)+a n^{2 / 3}$.

This equation can be considered as the reversible work of building a $(n, m, p)$ cluster involving four steps:

1. Formation of $n$ pairs of bulk AgI-the first term on the rhs of Eq. (3);

2. Addition of $m$ excess iodide molecules to the clusterthe second term;

3. Addition of $p$ excess $\mathrm{Q}$ (PEI or DMF) molecules to the cluster, this contributes $p \mu_{\mathrm{Q}}^{\text {ins }}(n)$, with $\mu_{\mathrm{Q}}^{\text {ins }}(n)$ the chemical potential of PEI (or DMF) as part of a cluster.

4. Surface term $a n^{2 / 3}$ : this term takes into account the interactions of a $(n, m, p)$ cluster with the electrolyte (after being removed from the bulk excess solid).

Combining the above equations leads to the size distribution of clusters of category $(n, m, p)$

$$
\begin{aligned}
c_{n, m, p}= & v_{S}^{-1}\left(f_{\mathrm{Q}} c_{\mathrm{Q}} e^{\Delta \mu_{\mathrm{Q}}^{\mathrm{ins}}(n)}\right)^{p} \\
& \times\left(f_{\mathrm{I}^{-}} c_{\mathrm{I}^{-}} e^{\Delta \mu_{\mathrm{I}^{-}}^{\mathrm{ins}}(n)}\right)^{m} e^{-a n^{2 / 3} / k T} .
\end{aligned}
$$

In this equation, $c_{n, m, p}=x_{n, m, p} / \nu_{S}$ is the (molar) concentration of the clusters of category $(n, m, p)$ and $v_{S}$ is the (molar) volume of the solvent (for water, $v_{S}^{-1} \approx 55.56 \mathrm{M}$ ). We have defined $\Delta \mu_{i}^{\text {ins }}(n)=\left(\mu_{i}^{0}-\mu_{i}^{\text {ins }}(n)\right) / k T$ as the difference in chemical potential between (a mole of) $i=$ iodide, PEI, or DMF in solution and in a cluster. This term takes into account that upon formation of an $(n, m, p)$ cluster, $m$ iodide ions and $p$ molecules of $\mathrm{Q}$ are transferred from solution to the solid cluster.

In general, the cluster composition may depend on the size, but as no information regarding such dependence is available, we pragmatically write the size distribution as

$c_{n}=b e^{-a n^{2 / 3} / k T}$,

where the "prefactor" $b$ depends on cluster composition, and therefore on the iodide and Q (PEI or DMF) concentration in solution. The dependence of $b$ on $n$ is a priori more complicated than in the system without PEI or DMF [1], but is still expected to be weak compared to the exponential dependence, i.e., the second term on the rhs of Eq. (A.5).

\section{Appendix B. Data analysis}

The values of $a$ in Eq. (A.5) were obtained as described in the main text. The values of $b$ cannot simply be extracted from the absorption spectrum, since the absolute optical absorption of the clusters is not precisely known. Absorption spectra of the samples were measured relative to samples without silver and PEI or DMF, but these samples still have a composition slightly different from that of the electrolyte in which the clusters are dispersed. In Ref. [1], the values of 
$b$ were obtained from the solubilities of silver in the aqueous phase. However, for the systems with PEI, these solubilities could not be determined. Therefore, the values of $b$ were extracted from the intercepts of plots of the $\ln A$ versus $n^{2 / 3}$, see Eq. (5). The value of the molar extinction coefficient of the clusters was assumed the same as reported in [1]. For the systems with 10,50 , and $100 \mathrm{mM}$ PEI, the intercepts $(\ln (\varepsilon l b))$ are $3.2,3.8$, and 3.9. This leads to values of $b=2.5 \times 10^{-4}, 4.5 \times 10^{-4}$, and $4.9 \times 10^{-4}$, respectively.

For the systems with dimethylformamide (DMF), the values of $b$ were obtained from the solubilities of silver as determined from the masses of the (dry) excess phases. Assuming that all silver is dispersed in the form of clusters (i.e., the number of free silver ions is much smaller than the amount of silver in the clusters), the solubility of silver is given by $c_{\mathrm{Ag}}=\sum_{n=1}^{\infty} n c_{n}=b \sum_{n=1}^{\infty} n e^{-a n^{2 / 3} / k T}$. In principle the values of $b$ can now be extracted from this equation using the solubilities $c_{\mathrm{Ag}}$ as well as the values of $\sum_{n=1}^{\infty} n e^{-a n^{2 / 3} / k T}$ that were calculated numerically.

The concentrations of silver in the solutions are $c_{\mathrm{Ag}}=$ $0.43,0.48,1.25$, and $0.57 \mathrm{M}$ for the systems with 0.05 , $0.3,0.5$, and 1.5 M DMF, respectively. The values of $\sum_{n=1}^{\infty} n e^{-a n^{2 / 3} / k T}$ are $1.66,2.13,2.25$, and 2.38 for the values of $a$ that correspond to $0.05,0.3,0.5$, and 1.5 M DMF. This leads to values of $b=0.26,0.22,0.56$, and 0.24 , respectively.

Comparing the values of the intercepts of plots of $\ln A$ versus $n^{2 / 3}$ (see Eq. (5)) being equal to $\ln (\varepsilon l b)$, i.e., 3.93, $3.55,3.69$, and 4.02 for systems containing $0.05,0.3,0.5$, and 1.5 M DMF with those of $b$ immediately gives an or- der of magnitude estimate of the molar extinction coefficient of the clusters. This value is $10^{2}-10^{3} 1 / \mathrm{mol} \mathrm{cm}$. This is a small value compared to the system without adsorbing species other than iodide [1], where it was found to be on the order of $10^{5} \mathrm{l} / \mathrm{mol} \mathrm{cm}$. Although we do not rule out that adsorbing species alter the extinction coefficient of the particles, we believe that the main cause for the apparent small value lies in the model. Either the value of $b$ or the cluster interfacial tension or both may depend on cluster size, while Lambert-Beer's law is not expected to apply in the concentration range of clusters being roughly $10^{2}-10^{3}$ times larger than in [1].

\section{References}

[1] I.L. Mladenovic, W.K. Kegel, P. Bomans, P.M. Frederik, J. Phys. Chem. B 107 (2003) 5717.

[2] E.J.W. Verwey, J.T.G. Overbeek, Theory of Stability of Lyophobic Colloids, Dover, New York, 1999.

[3] W.K. Kegel, J.T.G. Overbeek, H.N.W. Lekkerkerker, in: P. Kumar, K.L. Mittal (Eds.), Microemulsions, Fundamental and Applied Aspects, Dekker, New York, 1999, p. 13.

[4] A.E. Nielsen, O. Sohnel, J. Cryst. Growth 11 (1971) 233.

[5] P. Mulvaney, Colloids Surf. A 81 (1993) 231.

[6] F. Gaizer, P. Sipos, J. Lazar, Polyhedron 13 (1994) 855.

[7] S.V. Gaponenko, Optical Properties of Semiconductor Nanocrystals, Cambridge Univ. Press, Cambridge, UK, 1998.

[8] C. Berry, Phys. Rev. 161 (1967) 848.

[9] L.A. Kochanova, N.P. Fedoseeva, V.M. Kuchumova, A.V. Pertsov, P.A. Rebinder, Coll. J. USSR 35 (1973) 779.

[10] E.L. King, H.J. Krall, M.L. Pandow, J. Am. Chem. Soc. 74 (1952) 3492. 